selenium content of cereals exhibits greater regional variations than that of dairy or meat products. Thus, the intake of young children is markedly influenced by the origin of their cereals. Processing may also decrease the selenium content of food-stuffs. Commercially available meals contain less selenium than home-made ones (30 ng/g versus $50 \mathrm{ng} / \mathrm{g}$ ). Data about the gradual increase of the selenium intake during later childhood are scanty. In healthy adults the selenium intake ranges from about $20 \mu \mathrm{g} /$ day in New Zealand to $326 \mu \mathrm{g} /$ day in Venezuela, or from $11 \mu \mathrm{g} /$ day in selenium deficient areas in China to $4990 \mu \mathrm{g} /$ day in seleniferous areas.

Because of rapid growth children are more prone to selenium-deficiency states than adults. Groups at risk are besides formula-fed infants, children from selenium-poor areas (New Zealand, Finland and China), patients on parenteral nutrition or on semisynthetic diets. Reduced selenium states were first observed in patients with kwashiorkor. Selenium supplementation was reported to ameliorate the clinical condition of those patients from Guatemala. Selenium supplementation was also tried in patients with muscular dystrophy of Duchenne type.

Recent reports from China have shown a causal relationship between Keshan disease and selenium deficiency (3). This endemic cardiomyopathy occurred mainly in children and women of child bearing age in a long montaneous belt reaching from northeast to southwest of China. First low hair selenium values $(<120 \mathrm{ng} / \mathrm{g})$ were detected in children from affected areas. The selenium content of blood $(21+10 \mathrm{ng} / \mathrm{ml}$ was as well reduced as the erythrocyte glutathione peroxidase activity. This low selenium state derived from the low selenium intake by grains grown on soils with little available selenium. Selenium supplementation lead to a complete prevention of the disease. In two adults and in $12 \mathrm{yr}$ old child on a self-selected selenium-poor diet a cardiomyopathy was published and muscle pain, tenderness and fingernail-bed anomalies in an $8 \mathrm{yr}$ old boy on total parenteral nutrition (6) In China, in Keshan disease but also in Kaschin-Beck disease, selenium deficiency seems to play an important part in the etiology or pathogenesis (7). Kasschin-Beck disease is an osteoarthropathy with a disturbance of the enchondral ossification and deformity of affected joints. The low selenium content in cereals from Kaschin-Beck disease-affected areas was occasionally discovered as rats fed with these cereals suffered from typical signs of selenium deficiency, liver necrosis and white muscle disease. It is unclear whether Keshan disease and Kaschin-Beck disease are purely selenium deficiency diseases or whether other nutritional environmental and infectious facm tors contribute to the pathogenesis.

Selenium requirement may be different under different nutritional environmental, genetic and health conditions. Dietetically treated patients with PKU do not suffer from cardiomyopathy although their selenium states are equally low as in chinese children from Keshan-affected areas. The food composition may cause this difference. Interactions between selenium and proteins or methionine, vitamin E, arsenic, silver, mercury, polyunsaturated fatty acids are reported in animals. The nature of selenium compounds present in natural foods and their bio-availability is far from being known. In adults contradictory results about selenite-selenium, selenomethionine, Se-wheat, Se-yeast, fish-Se were published.

In children with reduced selenium state on parentera nutrition or semisynthetic diets selenium supplementation with selenite-Se $(3 \mu \mathrm{g} / \mathrm{kg} / \mathrm{d}$, parenterally) or Se-yeast (135 $\mu \mathrm{g} / \mathrm{m}^{2} / \mathrm{d}$, orally) was shown to be very effective. The selenium content and glutathion peroxidase activity of plasma increased within days and also the platelet glutathione peroxidase activity. Platelets belong to the tissues with high selenium content and corresponding glutathionine peroxidase activity. Erythrocyte glutathionine peroxidase activity only shows a change after long-term supplementation. Long-term changes of selenium supply can be also monitored by head hair analysis. A selenium depletion quickly occurs in infants during rapid growth. In older children or repleted patients a reduced selenium intake needs months to show off in glutathione peroxidase activities of plasma, platelets or erythrocytes. At the moment we do not know the safe and adequate range of selenium intake in children. Do the formula-fed infants with low blood and hair selenium values already suffer from a manifest or marginal selenium deficiency state, which may induce long-term effects on their health? At which level selenium intoxification may occur, either acute or chronic or by diminishing the bioavailability of other essential food stuffs? Acute selenium poisoning with unknown amounts of selenious acid or selenite was reported in three children, of whom one died 3 hours later. All three suffered besides the garlic odour from uncharacteristic symptoms, like hypersalivation, vomiting, diarrhoea, restlessness, arrhythmia. Plasma selenium was 20 times normal and urine selenium 100 times normal (5).

Selenium has a rather narrow therapeutic range. Supplementations should be performed only if necessary and carefully monitored to avoid intoxications or imbalances with other nutritional components.

References:

1) Bayliss PA et al 1985 Biol Trace Elem Res 7, 53

2) Casey E et al 1982 Arch Environ Health 37: 133

3) Chen $x$ et al 1980 Biol Trace Elem Res 2: 91

4) Collip PJ, Chen SY 1981 New Engl J Med 304, 1305

5) Lombeck I et al (in press) 1986 Eur J Ped

6) Kien CL, Ganthex H.E 1983 Am J Clin Nutx 37: 319

7) Dongxu M, Peking, 3.5.1984, III. Int. SIMP Se.

8) Westermarck T 1977 Acta Pharmacol Toxicol 41, 121

9) Widdra $S$, Lombeck I: unpublished results

\section{PRENATAL NEUROLOGY}

Heinz F.R. Prechtl

Department of Developmental Neurology, University of Groningen, Groningen, The Netherlands

The relationship between obstetrical complications and neurological sequelae has frequently been investigated by correlating perinatal data with neurological follow-up data.

There is always a considerable time lag between these two data sources. It is now possible to assess the condition of the fetal nervous system during the acute or chronic phase of obstetrical complications without such delay through ultrasound observations of the fetus.

Fetal movement patterns are from their first onset (8-14 weeks p.m. age) distinct and coordinated and resemble patterns known from the newborn infant. They are endogenously generated and their incidence of occurrence follows a developmental course during pregnancy, which is specific for each individual movement pattern. They first appear following a characteristic time table (1). Quantitative assessment of the different movement patterns reveals an age specific motor repertoire in all longitudinally observed fetuses (2). 
In fetuses with gross brain malformations such as anencephaly, severely distorted movements have been found. Not only is the quality of movements abnormal but also their temporal sequence. Frequently, the different movement patterns are no longer distinguishable but merge in a "motor cacaphony" (3) In growth retarded fetuses the quality of movement patterns is changed although they are still recognizable as such. They become monotonous and lack the normal subtle fluctuations in speed, force and amplitude. Similar to the case in neurologically abnormal preterm infants (4), abnormal fetal movements can be reliably recognized by independent observers (5).

Changes in the quantity of fetal movements is a less sensitive indicator as this only decreases markedly in very serious conditions, i.e. terminal states. The ultrasound observation of fetal movements is a promising approach to the neurological assessment of fetuses exposed to a compromizing condition.

1. Vries JIP de, Visser GHA, Prechtl HFR 1982 The emergence of fetal behaviour. I. Qualitative aspects. Early Hum Develop 7: 301-322

2. Vries JIP de, Visser GHA, Prechtl HFR 1985 The emergence of fetal behaviour. II. Quantitative aspects. Early Hum Develop 12: 99-120

3. Visser GHA, Laurini RN, Vries JIP de, Bekedam DJ, Precht1 HFR 1985 Abnormal motor behaviour in anencephalic fetuses. Early Hum Develop 12: 173182

4. Prechtl HFR, Nolte R 1984 Motor behaviour of preterm infants. "Continuity of neural functions from prenatal to postnatal life" (Prechtl HFR ed) Blackwell Scientific Publications Ltd, Oxford, England, Clin Develop Med 94: 79-92

5. Bekedam DJ, Visser GHA, Vries JJ de, Prechtl HFR 1985 Motor Behaviour in the growth retarded fetus. Early Hum Develop 12: 155-166

LANGUAGE DEVELOPMENT DURING THE FIRST FIVE YEARS OF LIFE IN TERM AND PRETERM CHILDREN

Remo H. Largo

Kinderspital, CH-8032 zürich, Switzerland

While the overall development of premature children has been studied extensively by means of developmental and intelligence testing (review article see 8 ), language development has been underinvestigated in this population (6). Since prematurity is a major risk factor leading to developmental disturbances in postnatal life (5), and delayed early milestones of language development have been postulated as a sensitive indicator of developmental disability (e.g. 1), language development in prematurely born children is of particular interest.

Prematurely born children are reported to be mildly delayed in language development when compared with full-term populations (e.g. 7). One of the possible causes for this delay might be an organic cerebral lesion. None of the past studies on language development in preterm infants has the portion of neurologically impaired children included and the degree of neurological impairment taken into consideration. In our study, language development was investigated in neurologically unimpaired and impaired preterm infants separately (3). The former were mildly delayed in their early language development, performed slightly less well in the Illinois test of psycholinguistic abilities and showed more articulation defects at age 5 in comparison with the term group. This delay was comparable to that observed in the early development of locomotion in neurologically unimpaired preterm infants (2). Infants with mild to moderate cerebral palsy showed a consistent delay in their language development and had more articulation problems at age 5 .

Language development in preterm infants might be impaired because they are genetically slightly deviant and/or experienced adverse events in early pregnancy. Complications during later pregnancy, delivery or the neonatal period may lead to impaired cerebral functioning, resulting in a language delay. Finally, unfavourable environmental conditions may have a negative effect on language development. Multiple minor malformations, possibly indicating mild genetic deviances or adverse events in early pregnancy, were significantly more frequent in the preterm than in term infants. However, a significant relationship between the frequencey of minor malformations and language development could not be established in the preterm group. Minor genetic deviancies and/or minor adverse events in early pregnancy did not seem to have a strong enough impact on the developing brain to delay language development significantly.

The perinatal optimality score (POS), a measure for optimality of pregnancy, birth and the neonatal period, was significantly negatively correlated with language development. Our results indicate a more pronounced effect of adverse perinatal events on motor functioning than on the processes of language comprehension and expression. Gestational age and birth weight were consistently negatively correlated with language performance, a finding which could only partially be explained by the relationships between gestational age, birth weight and POS. It is tempting to speculate that, before term, presently unknown factors, e.g. of metabolic origin, might have a negative impact on early extrauterine brain development.

Socio-economic status (SES) and birth order had an age related influence on language development in term and much less in preterm children. In contrast to previous studies (e.g. 7, 9) the significance of SES in our preterm group was maxginal, while perinatal factors exerted a considerable effect on their language development. We assume that the most homogeneous and better socio-economic conditions in our country than for example in the United States or England led to our finding that perinatal factors have a greater impact on language development than has previously been reported.

Acknowledgements: The collaboration of Dr L Molinari, Dr M Weber, Dr L Comenale-Pinto is gratefully acknowledged.

This work was supported by the Swiss National Science Foundation Nr 3988-0.84.

References:

1. Coplan J et al 1982 pediatrics 70:677

2. Largo R H 1985 Develop Med Child Neurol 27:183

3. Largo et al 1986 Develop Med Child Neurol (in press)

4. PrechtI HFR 1980 Early Hum Develop 4: 201

5. Reed DM 1977 Baltimore: Urban\&Schwarzenberg

6. Rocissano L, Yatchmink Y 1983 Child Development $54: 1229$

7. Siegel LS 1982 Child Development 52:545-557

8. Stewart AL 1981 Lancet $i: 1038$

9. Wright NE 1983 Brit J Disord Communic 18:187 